\title{
SCALING UP THE USE OF ANTIRETROVIRALS IN THE PUBLIC SECTOR: WHAT ARE THE CHALLENGES?
} Helen Schneider, Centre for Health Policy, School of Public Health, University of the Witwatersrand, fohannesburg
James McIntyre, Perinatal HIV Research Unit, Chirs Hani Baragwanath Hospital, Sowveto, Gauteng

On 1 August 2003 the Wits School of Public Health and Perinatal HIV Research Unit hosted a 1-day seminar on the challenges to scaling up access to antiretroviral therapy (ARV or ART) in South Africa.

The meeting was attended by 130 people, representing a wide variety of backgrounds and including such diverse role players as people involved in pilot programmes successfully delivering antiretrovirals in poor communities (including a neighbouring country) and those involved in the roll-out of the mother-to-child transmission prevention (PMTCT) programme and the national tuberculosis programme, and the implementation of the Termination of Pregnancy Act.

Challenges were discussed from all these perspectives during the course of the seminar, with the purpose of specifically addressing the health systems challenges. The seminar deliberately did not address two key aspects of the scaling up procurement: provision of affordable drugs, and the mobilisation of financial resources for the implementation of a programme, as these were being debated in other fora.

\section{SUMMARY OF PROCEEDINGS}

Highly active antiretroviral therapy (HAART, also referred to as ART or ARVs) makes a dramatic difference to the survival and health of people living with HIV. At present only about 1000 public sector users in South Africa have access to ARVs through a series of small-scale projects across the country. Greater access to ARVs could change the lives of millions of people. It could also bring with it large new investments in the health system that, if properly planned, could address systemic weaknesses as well as strengthen less resourced areas. It is imperative that these investments

This article reflects the outcomes of a Seminar hosted by the School of Public Health and Perinatal HIV Research Unit, University of the Witwatersrand, on Friday 1 August 2003.

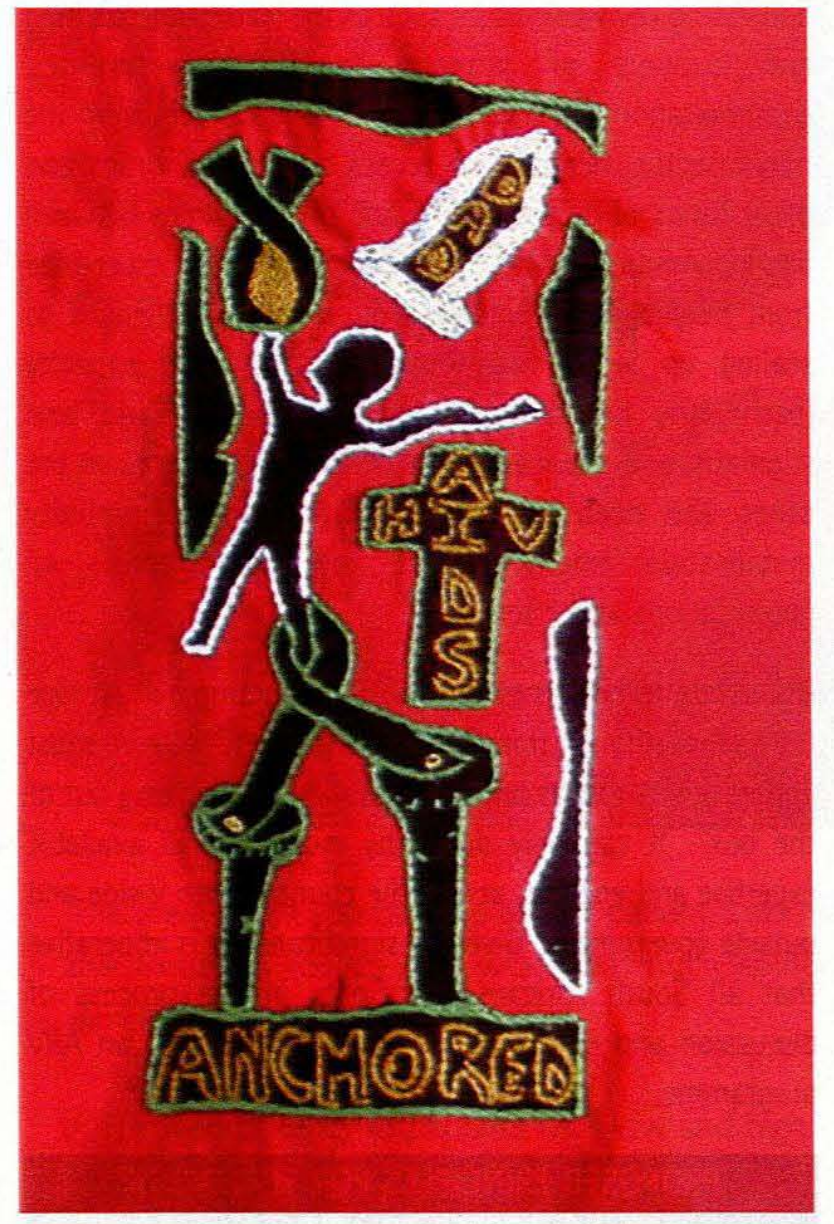

are implemented in a manner that benefits the health system as a whole and builds equity. A programme of ARV access also has to grapple with the challenge of ensuring that people take drugs several times a day, every day, for the rest of their lives. Poor adherence rapidly leads to the emergence of drug resistance and treatment failure at the individual level. If resistance develops on a wide scale, this will have broader public health implications. Obligations on the part of individuals to adhere have to be matched by obligations on the part of the health system to ensure continuous access to uninterrupted supplies of drugs, skilled providers and laboratory support able to maximise the safety and efficacy of drugs, and a supportive environment for adherence. Unfortunately, the margin for learning through failure is narrow. 
A number of projects have shown that these challenges can be confronted on a small scale within the public sector, such that the benefits of ARVs far outweigh the risks associated with them. South Africa has a health care infrastructure that has proved that it is capable of providing chronic disease care, most notably for tuberculosis. Scaling up access to ARVs through this system is not outside the bounds of imagination. However, the TB programme and the implementation of the Termination of Pregnancy Act and PMTCT programme provide evidence highlighting the fact that a programme of universal access to ARVs will require performance from our health system at a level and scale far higher than at present. It means addressing key weaknesses, such as the inadequate staffing and support of primary health care facilities, demoralisation and flight of health care workers from the public sector, inequities in access to facilities, drugs and other forms of infrastructure, and poor relations between users, communities and the health system. The experience from neighbouring Botswana is that the challenges to scaling up lie in two areas: health system capacity (particularly human resources) on the one hand, and stigmatisation and inadequate demand for services on the other. The need for communication and community mobilisation emerged as a key theme across all presentations at the seminar.

Ultimately, to be successful, an ARV programme must face the complexity of transforming relationships - it must appreciate its role as one of innovation, of 'thinking out of the box.' It can only do so by mobilising all available expertise and energies across the country, both inside and outside government. Given its significance, it is imperative that all South Africans participate in the process of discussion and debate about how to implement an ARV programme.

\section{PROPOSED PRINCIPLES FOR SCALING UP}

\section{ACCESS TO ARVs}

1. A policy on widespread access to ARVs can only succeed if it fully mobilises the existing health system. It should form an integral part of the continuum of HIV care through the public primary health care and hospital system, coordinated by district, provincial and national management structures. This does not preclude a degree of 'exceptionalism' and vertical programme arrangements, under very specific conditions; nor does it exclude the possibility of co-ordinated action between public and private sectors where this is deemed relevant at local level.

2. An ARV programme integrated into the health system, however, will not succeed if it is regarded as a simple 'add${ }^{\prime} n^{\prime}$ to the multiple functions and activities already performed by the health system. Additional resources must be provided, particularly in the range and numbers of skilled personnel at facility level, but also in support systems. New ways of managing existing resources need to be developed.

3. The massive additional investment in health systems brought about by an ARV programme provides a unique opportunity to strengthen the health system as a whole. Improvements in systems, such as drug supplies, access to laboratory services, referral, and staff training and support, should be structured to strengthen quality and access for all health conditions. An ARV programme should be seen as an opportunity to address fundamental problems, such as the public sector human resource crisis and provider attitudes and values. It should not be implemented at the cost of other essential health programmes.

4. An ARV programme should not deepen the inequities in our health system. Ultimately, it must be judged by whether it succeeds in reaching the most remote and disadvantaged areas of the country. This may mean special measures to build the 'capacity to benefit' from an ARV programme in disadvantaged areas. This would require investment in basic systems and infrastructure upon which a continuum of HIV care, including ARVs, can be built.

5. Existing public health and HIV treatment initiatives in South Africa provide useful models for the design of an ARV programme. Experiences with the procedures, systems and infrastructure of the national TB control programme can inform an ARV programme. They include standardisation of treatment, registers, monitoring and evaluation processes and the particular combination of vertical support and horizontal implementation. Existing ARV projects suggest that an ARV programme integrated into a broader HIV care treatment service can be configured in the same way as other chronic disease programmes, as nurse-based follow-up with adequate doctor support.

6. Alternatives to the main approach to tuberculosis care, namely 'directly observed therapy', however, are needed if the stringent adherence requirements of ARVs are to be achieved. The evidence from pilot projects is that high levels of adherence stem from a new kind of contract between providers and clients. This contract is premised on very high levels of understanding or treatment literacy on the part of users. It requires the establishment of explicit support systems for users and community mobilisation and advocacy processes that promote the rights of people living with HIV/AIDS. The responsibility for adherence is given to the client him/herself, but occurs within a clear framework of empowerment, a period of treatment preparedness and the building of trusting relationships with providers. This is very different to the traditional paternalistic and passive relationship between health care workers and patients, and 
making the change represents the key innovation challenge of an ARV programme. Central to this relationship are the front-line providers, who have to be won over to the purpose, content and process of an ARV programme.

7. How an ARV programme is implemented is as important as the what of the programme. To build the levels and scale of performance required of an ARV programme necessitates an innovative approach to implementation. Task teams developing guidelines and procedures at national level alone will not achieve the rapid buy-in and change required at all levels of the system. Political management, in the sense of actively building common visions, is as important as technical design. We therefore propose a set of sub-principles to govern the implementation process:

- The planning process should be as inclusive as possible. It is important that the voices of all stakeholders with insight and an interest in the implementation of an ARV programme be represented in defining the vision and principles of the programme. These include line and programme managers at national, provincial and local/district levels; clinicians and others involved in pilot ARV programmes or who have shown that they can provide good quality HIV care in the public sector; front-line providers, NGOs and activist groupings involved in community mobilisation; and researchers who can support monitoring and evaluation. Donors and external consultants, while clearly important stakeholders, should not dominate the process. The roles and responsibilities of all these role players need to be discussed and agreed upon.

The process of implementation needs to be supported by a rigorous and creative communication strategy aimed at preparing and ensuring the buy-in of all who will be implicated in the process of implementation. Its aim would be to develop a common vision and understanding, as the foundation for action.

a Top-down planning should be combined with bottomup processes that harness local energy and stimulate initiative. The need for defining clear outcomes and standardisation must be balanced with flexibility in programme implementation at local level. It also requires designing an implementation strategy that involves constant engagement with the periphery in iterative processes of learning and problem solving, of learning by doing.

- District and facility actors are much more likely to take up the challenge of an ARV programme if they can witness it being done in other districts and by other actors. Horizontal processes of interaction will lead to more effective learning than instructions from the top. A key aspect of implementation must be to promote networking between actors already involved in HIV treatment programmes and those wishing to develop programmes in their areas.

An incremental, step-wise process of building capacity for ARVs needs to be defined. Building blocks such as the establishment of a good follow-up, 'wellness' service for people with HIV, evidence of community mobilisation and support groups, voluntary counselling and testing and laboratory infrastructure need to be spelt out Support strategies need to involve not only training but also mechanisms for developing infrastructure and systems at facility and district levels.

ahile certain aspects of implementation need to be standardised, local people should be given some choice and flexibility in the content and pace of implementation. This includes front-line providers, users and community members who are the ultimate implementers of policy. We propose, in the first instance, a process of voluntary accreditation, in which facilities and their community and district level counterparts apply for the right to distribute ARVs. The onus will rest on them to prove their ability to do so and will be formally assessed through an accreditation process where clear criteria are spelt out. These districts could then form learning partnerships with others in the process of horizontal learning described above. Obviously, such a process would require co-ordination and management to ensure that equity is maintained.

An ARV programme is one necessary and important component of a wider social response to HIV/AIDS in South Africa. The HIV epidemic represents both a crisis and an opportunity for the country - currently in the midst of profound and dynamic transformation. In this context, a commitment to scale up the introduction of ARVs must be viewed alongside a measured and multidimensional approach to HIV - one founded on a renewed commitment to prevention and to a broader social response to the structural conditions that influence vulnerability to HIV.

\section{FINAL COMMENT}

South Africa has the potential to generate a model approach to the introduction of ARVs that has relevance and application to the wider sub-Saharan region. As unprecedented external funds are made available to support and facilitate this process, effective co-ordination and providing clear direction around the care components of the ARV-health systems approach is essential. In this context, there is a need to balance effective partnerships and the generation of creative responses on the ground with a comprehensive vision for the systems required to sustain such an ambitious intervention in the long term. 